\title{
TIM-1 acts a dual-attachment receptor for Ebolavirus by interacting directly with viral GP and the PS on the viral envelope
}

\author{
Shuai Yuan ${ }^{1,2}$, Lei Cao ${ }^{1,3}$, Hui Ling ${ }^{4}$, Minghao Dang ${ }^{1}$, Yao Sun ${ }^{1}$, Xuyuan Zhang $^{1}$, Yutao Chen ${ }^{1}$, Liguo Zhang ${ }^{1}$, \\ Dan $\mathrm{Su}^{3}$, Xiangxi Wang $^{1 凶}$, Zihe Rao $^{1 \bowtie}$ \\ ${ }^{1}$ National Laboratory of Macromolecules, Institute of Biophysics, Chinese Academy of Science, Beijing 100101, China \\ 2 University of Chinese Academy of Sciences, Beijing 100049, China \\ ${ }^{3}$ State Key Laboratory of Biotherapy/Collaborative Innovation Center of Biotherapy, West China Hospital, Sichuan University, \\ Chengdu 610041, China \\ ${ }^{4}$ College of Biotechnology, Tianjin University of Science and Technology, Tianjin 300457, China \\ $\bowtie$ Correspondence: wangxiangxi.wang@gmail.com (X. Wang), raozh@xtal.tsinghua.edu.cn (Z. Rao) \\ Received September 10, 2015 Accepted September 30, 2015
}

\begin{abstract}
Ebolavirus can cause hemorrhagic fever in humans with a mortality rate of $50 \%-90 \%$. Currently, no approved vaccines and antiviral therapies are available. Human TIM1 is considered as an attachment factor for EBOV, enhancing viral infection through interaction with PS located on the viral envelope. However, reasons underlying the preferable usage of hTIM-1, but not other PS binding receptors by filovirus, remain unknown. We firstly demonstrated a direct interaction between hTIM-1 and EBOV GP in vitro and determined the crystal structures of the Ig V domains of hTIM-1 and hTIM-4. The binding region in hTIM-1 to EBOV GP was mapped by chimeras and mutation assays, which were designed based on structural analysis. Pseudovirion infection assays performed using hTIM-1 and its homologs as well as point mutants verified the location of the GP binding site and the importance of EBOV GP-hTIM-1 interaction in EBOV cellular entry.
\end{abstract}

KEYWORDS Ebolavirus, viral entry, glycoprotein, receptor, interaction

Electronic supplementary material The online version of this article (doi:10.1007/s13238-015-0220-y) contains supplementary material, which is available to authorized users.

\section{INTRODUCTION}

Ebolavirus (EBOV) and Marburgvirus, belonging to the Filoviridae family, cause hemorrhagic fever during the course of their infections in humans. Alarmingly, infections caused by these viruses have a high mortality rate of $50 \%-90 \%$ (Dolnik et al., 2008). In 2011, human T-cell immunoglobulin and mucin domain protein 1 (hTIM-1), previously implicated as a receptor for hepatitis A virus (Silberstein et al., 2003; Feigelstock et al., 1998; Wang et al., 2014), was reported as a receptor for EBOV and Marburgvirus (Kondratowicz et al., 2011). Recent studies indicate that hTIM-1, with some other but not all phosphatidylserine (PS) binding receptors, functions as a common attachment factor for a range of enveloped viruses, including filoviruses, flaviviruses, HIV, etc., through direct interaction with PS of the viral envelope $(\mathrm{Li}$ et al., 2014; Moller-Tank et al., 2013; Meertens et al., 2012; Jemielity et al., 2013). Interestingly, hTIM-1 can mediate uptake of viruses independent of the glycoproteins (MollerTank et al., 2013; Jemielity et al., 2013; Takada et al., 1997). However, recently hTIM-1 was shown to interact with NPC1, a fusion receptor for filovirus. This interaction was important for the entry of EBOV into host cell (Côté et al. 2011; Kuroda et al., 2015), thus raising an important question on whether hTIM-1 functions as an attachment factor or it is a bona fide receptor for EBOV.

TIM family proteins exhibit a classical type I membrane protein structure with an $\mathrm{N}$-terminal immunoglobulin variable Ig-like (Ig V) domain, a heavily O-linked-glycosylated mucinlike domain (MLD) and a short C-terminal cytoplasmic tail 
(Freeman et al., 2010). Three human TIM proteins (hTIM-1, 3 and 4) and eight mouse TIM proteins (mTIM 1-8) have been identified so far. hTIM-1, 3 and 4 are considered direct orthologs of mTIM-1, 3 and 4, respectively (Kuchroo et al., 2003). More importantly, except for mTIM-2, the Ig V domains of all TIM proteins were predicted to contain a conserved PS binding site (Santiago et al., 2007). This is important in context of cellular entry of virus because PS is exposed on the membranes of various enveloped viruses and is known to play an important role in mediating viral entry (Mercer and Helenius, 2008; Soares et al., 2008). Intriguingly, PS receptor usage by different enveloped viruses for entry into host cells differs significantly, which might reflect distinct mechanisms involving additional interactions between viruses, PS receptors and other host factors (Moller-Tank et al., 2013; Jemielity et al., 2013). EBOV preferably utilizes hTIM-1, not other TIM proteins, to mediate the entry efficiently, which suggests that hTIM-1 probably has a direct or indirect interaction with EBOV glycoprotein (GP).

The GP of EBOV is composed of a trimer of disulfidebonded GP1/GP2 heterodimers, which mediates receptor (s) binding, internalization, penetration and fusion with hostmembranes (Takada et al., 1997; Wool-Lewis and Bates, 1998; Lee et al., 2008). GP1 contains the receptor binding domain (RBD), which is used to interact with its cellular receptor(s), while GP2 mediates virus/host membrane fusion events (Kuhn et al., 2006; Brindley et al., 2007). The GP is necessary and sufficient to mediate entry of EBOV or Ebola pseudovirion into plasma of host cells (Hunt et al., 2012).

Although methods used to study cellular entry of EBOV that rely on incorporation of Ebola GP onto surrogate viruses (HIV-1 pseudotypes) renders the study of EBOV entry easier, these methods do not exclude the possibility of a role for the PS located on the pseudovirion membrane in the process of viral entry into host cell. A structural view of the virus-receptor interaction or at least of the receptor would be more instructive about whether additional factors are involved in the attachment step. To clarify the role of hTIM-1 in cellular entry of EBOV, we first studied the interaction of hTIM-1 with GP of EBOV in vitro. We show that the $\lg V$ domain of hTIM-1, but not other TIM family members, interacts directly with the GP and more specifically with the $\mathrm{RBD}$ of EBOV in vitro. Interestingly, the interaction was moderately inhibited by PS in a dose dependent manner. Furthermore, we determined the crystal structures of the Ig $\mathrm{V}$ domains of hTIM-1 and hTIM-4. Using the crystal structure as a guide we designed hTIM1-hTIM4 chimeras and studied their binding to EBOV GP to map the location of the GP binding site. Point mutants of hTIM-1 identified residues critical for GP-binding. Pseudovirion assays using point mutants of hTIM-1 confirmed the importance of the interaction interface in EBOV infection.

\section{RESULTS}

\section{Preparation and characterization of $\mathrm{Ig} \mathbf{V}$ domains} of hTIM1, 3 and 4

Ig V domains of hTIM-1, 3 and 4 with a C-terminal 6× His tag were produced by using bacterial expression system as inclusion bodies. Ig $\mathrm{V}$ domains were successfully recovered from inclusion bodies by employing a de-naturation and refolding protocol. The proteins were further purified by $\mathrm{Ni}$ NTA affinity chromatography and size exclusion chromatography (See "MATERIALS AND METHODS"). SDS-PAGE and thermal stability analysis as well as PS binding assays verified that the $\lg \mathrm{V}$ domains of hTIM-1, 3 and 4 represent functional units, with good purities and stabilities (Fig. 1A-C). Given the importance of oligomeric state in determination of the signaling mechanism of cell-surface receptors, the solution behaviors of the hTIM-1, 3 and $4 \mathrm{lg} \mathrm{V}$ domains were examined. Sedimentation velocity experiments using an analytical ultra-centrifuge revealed that Ig $\mathrm{V}$ domains of hTIM1, 3 and 4 existed as a monomer in solution (Fig. 1D).

\section{hTIM-1 Ig V is the only TIM family protein that interacts with Ebola GP}

To explore the potential interactions between TIM family proteins and EBOV GP in vitro, Ig V domains of mTIM1-4 (purchased from Sino Biological Inc.), hTIM-1, 3, 4 and recombinant GPs of Ebola (Zaire and Bundibugyo strain) were prepared (Fig. S1) (See MATERIALS AND METHODS). Results of binding assays performed using SPR showed that amongst the proteins tested only hTIM-1 Ig V could bind EBOV GP. To verify the specificity of the binding, 4 different constructs of EBOV GPs (Zaire GP 1-501, 1-320, 1-308 with a His tag and Bundibugyo GP 1-308 with a Fc tag) were tested. These constructs of GP presented a similarly high binding affinity of 4.9-26.7 $\mu \mathrm{mol} / \mathrm{L}$ for $\mathrm{hTIM}-1$ (Fig. 2A-D). Bundibugyo GP showed the strongest binding affinity for hTIM-1 Ig V with a dissociation constant $\left(K_{d}\right)$ of about $4.9 \mu \mathrm{mol} / \mathrm{L}$, while GP and RBD from Zaire exhibited similar binding affinities for hTIM-1 Ig $\vee$ (Fig. 2B-D). Interestingly the interaction between EBOV GP and hTIM-1 Ig V can be moderately inhibited by $P S$ in a dose dependent manner. A 100-fold (mol/mol) excess PS in PBST buffer reduced binding efficiency of $\mathrm{hTIM}-1$ to GP by $\sim 25 \%$ (Fig. 2E), indicating the possibility that the binding sites of hTIM-1 to GP and PS overlap partially or fully. Deglycosylated RBD showed a $\sim 50 \%$ reduction in binding affinity for $\mathrm{hTIM}-1$, indicating that glycans on GP participate, but are not critical for the binding with hTIM-1 (Figs. 2F and S2).

\section{Structures of the Ig V domains of hTIM-1 and hTIM-4}

We crystallized the Ig $\mathrm{V}$ domains of hTIM-1 and hTIM-4 and determined the structures at a resolution of $1.3 \AA$ and $2.3 \AA$, 
A

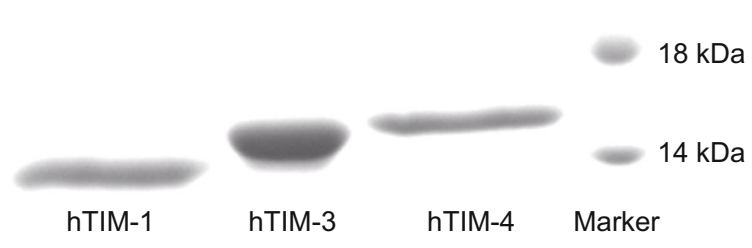

C

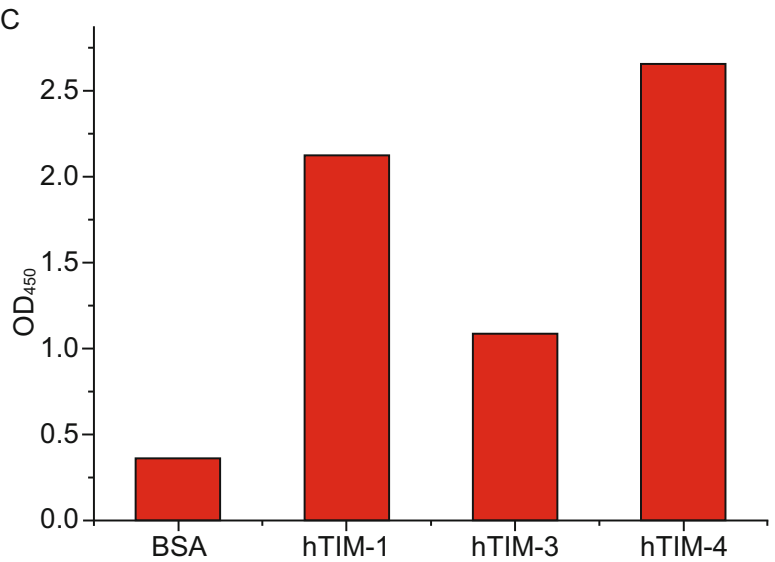

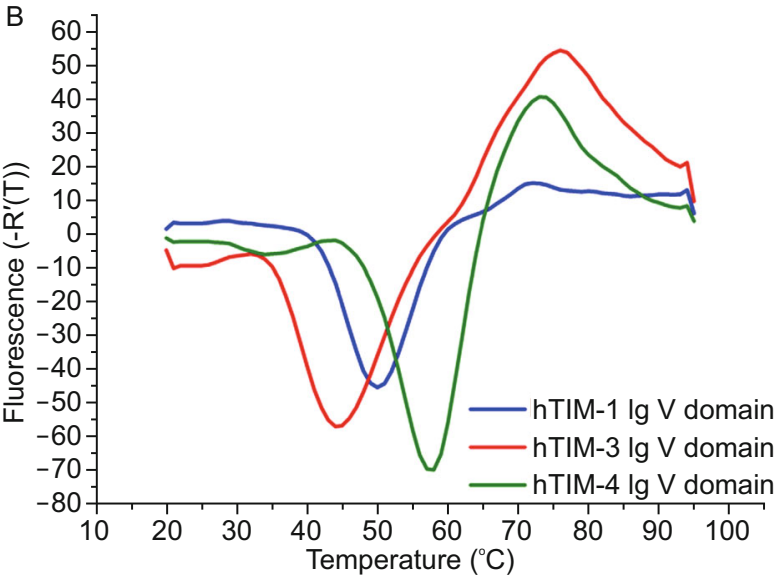

D

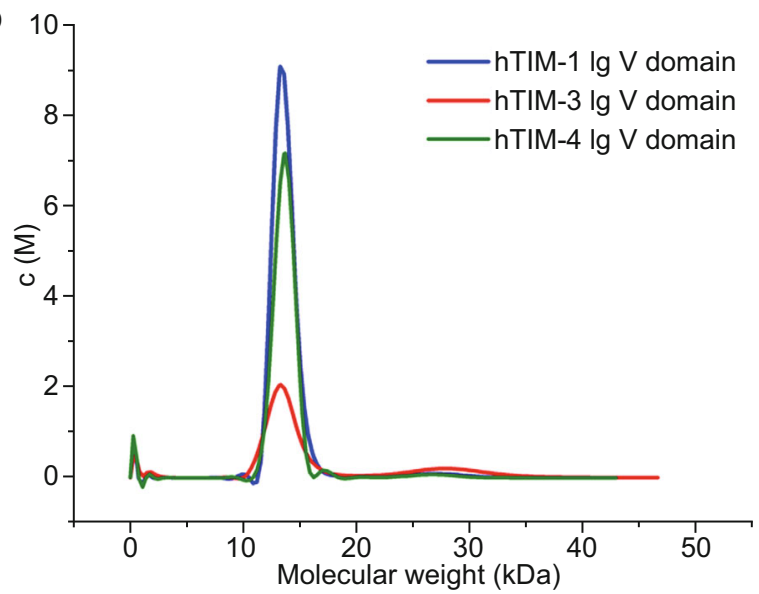

Figure 1. Characterization of recombinant Ig V domains of hTIM-1, hTIM-3 and hTIM-4. (A) SDS-PAGE analysis of Ig V domains of several human TIM proteins under reducing condition. (B) First derivatives of the fluorescence curves to measure the stability of $\mathrm{lg} V$ domains of several human TIM proteins. Human TIM lg V proteins were mixed with Sypro Orange dye and heated to indicated temperatures. Raw fluorescent signal increased as the dye bound to hydrophobic residues that were exposed from thermally destabilized proteins. (C) Binding of Ig V domains of several human TIM proteins to PS. Optical density (O.D.) was monitored at $450 \mathrm{~nm}$ to detect binding of human TIM proteins with Ig $\vee$ domains to PS liposomes immobilized on plastic plates (See MATERIALS AND METHODS). (D) Analysis of oligomerization state of $\mathrm{lg} V$ domains of several human TIM proteins in solution by sedimentation velocity experiments.

respectively, using the molecular replacement method (See MATERIALS AND METHODS and Table S1). While hTIM-1 has only one molecule in the asymmetric unit, there are eight molecules of essentially the same structure present in the asymmetric unit of hTIM-4. The Ig V domains of hTIM-1 and hTIM-4, exhibit two antiparallel $\beta$ sheets with the front and back faces formed by $A, G, F, C, C^{\prime}, C^{\prime \prime}$ and $B, E, D$ strands respectively (Fig. $3 A$ and $3 C$ ). The overall structure closely mirrors the structure of $\mathrm{mTIM}-4$ bound with PS. A hydrophobic cleft formed by CC' and FG loops, located at an equivalent position in hTIM-1 and hTIM-4 structures represents the putative PS ligand binding site (Fig. 3C). Residues known to interact with PS, including WFND (Santiago et al., 2007) of the FG loop, are conserved among hTIM-1, mTIM-1, hTIM-4 and mTIM-4 (Fig. 3A). The amine group of the Ser residue of PS interacts with the Asp and the fatty acid moiety of PS is likely to be stabilized by the hydrophobic side chains of Trp and Phe (Fig. 3C). While mTIM-2 lacks the WFND motif
(Fig. 3A) and therefore does not bind PS (Santiago et al., 2007), the aromatic residues, WF, of hTIM-3 and mTIM-3 are replaced with hydrophobic residues LM and IM, respectively. These substitutions lower the PS binding affinities of TIM-3 when compared to those of TIM-1 and TIM-4 (Figs. 1C and 3A). A striking structural observation that was confirmed by sequence analysis indicates that the $\lg \mathrm{V}$ domains of hTIM-1 and hTIM-4 share similarity with the N-terminal immunoglobulin-like domain of sialecsialo adhesin 1 (SnD1), hTIM-1 and hTIM-4 could be superimposed over the structure of SnD1 with an r.m.s. deviation of $2.3 \AA$ and $2.2 \AA$, respectively, (Fig. 3B and 3D). This resemblance suggests that hTIM-1 and hTIM-4 Ig $V$ domains might be able to bind carbohydrates using a siglec-like sialic acid binding motif. Three conserved residues (Trp 2, Arg 97 and Trp 106 in Siglec-1) together with a number of hydrophobic amino acids constitute sialic acid binding sites in all the siglecs (May et al., 1998). The putative PS binding clefts of hTIM- 1 and hTIM- 4 share the second and 
A

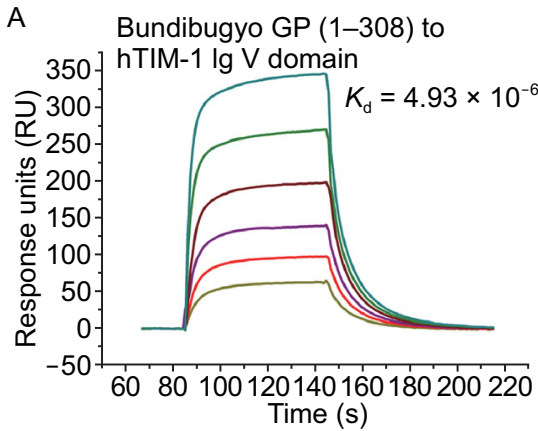

D

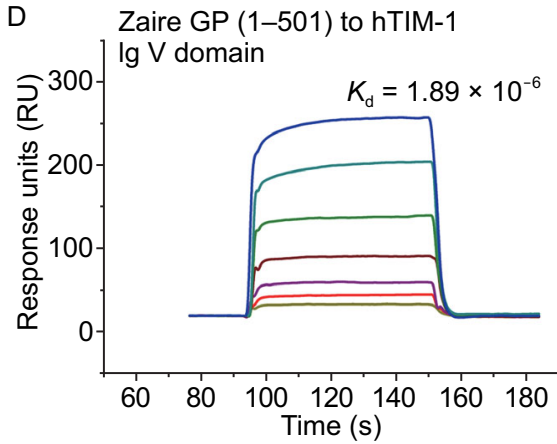

B

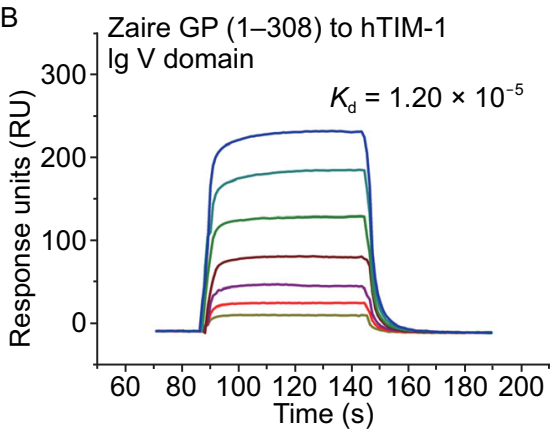

E

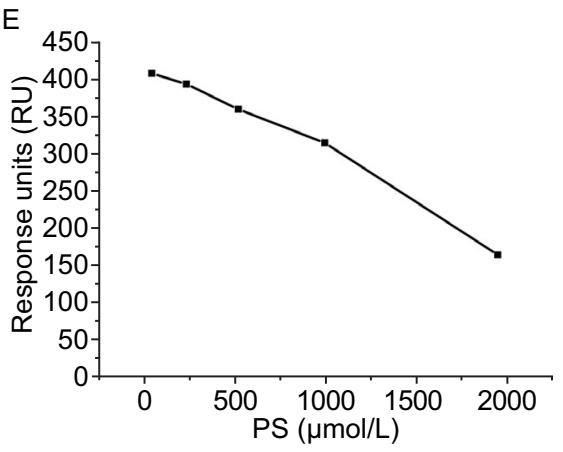

C 800 Zaire GP (1-320) to hTIM-1

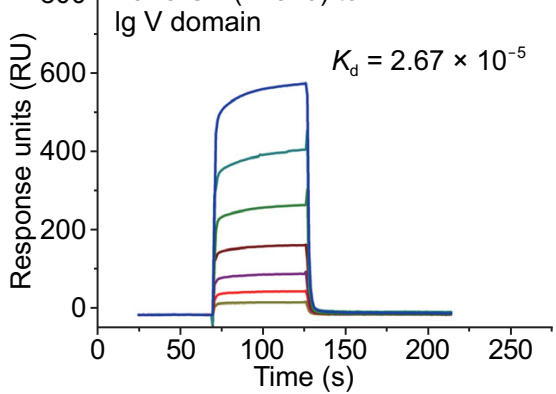

F 600 Deglycosylated Zaire GP (1-320)

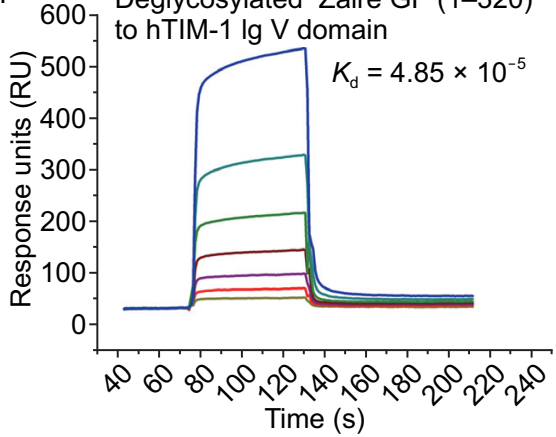

Figure 2. Surface plasmon resonance assay for characterizing the specific binding between hTIM-1 and EBOV GP. The profiles shown are as follows (A) Bundibugyo GP (1-308), (B) Zaire GP (1-308), (C) Zaire GP (1-320) and (D) Zaire GP (1-501) binding to hTIM-1 Ig V. (E) PS inhibited the binding between hTIM-1 and EBOV GP in a dose dependent manner. Response units were plotted against PS concentrations (See MATERIALS AND METHODS). (F) Binding of deglycosylated Zaire GP (1-320) to hTIM$1 \mathrm{lg}$ V. The binding affinities $\left(K_{\mathrm{d}}\right)$ values were calculated using a steady-state affinity model produced with BIAcore 3000 analysis software (BIA evaluation Version 4.1).

third (Arg and Trp) residue with siglecs. However the Trp is replaced by a Phe. Similar to other siglecs, hTIM-1 and hTIM4 use hydrophobic residues of the CC' loop to complete the binding site (Fig. 3B and 3D). Thus, our structural studies indicate that in addition to PS, hTIM-1 and hTIM-4 could possibly bind other ligands; in particular, carbohydrates.

\section{A unique groove adjacent to PS binding site in hTIM-1}

Despite sharing overall structural similarities with other $\lg \mathrm{V}$ domains of TIM family proteins, hTIM-1 Ig V domain exhibits unique features that may be essential for its distinct biological roles. Most notably, hTIM-1 possesses a significantly short BC loop that is the most variable structural feature amongst Ig $\mathrm{V}$ domains of the TIM family. In addition the residues of $B C$ loop carry smaller side chains when compared to other TIM family proteins (Fig. 3A). This structural feature coupled with the movement of FG loop of hTIM-1 away from the $\mathrm{BC}$ loop, creates a unique groove that merges with the PS binding cleft (Fig. 4). Two residues (Arg 110 and Arg 86 ) in hTIM-1 (while the counterparts are Pro and Gly, respectively in other TIM family members) render the two walls of the groove positively charged (Fig. 4). To find out why only hTIM-1 but not other TIM proteins bound EBOV GP, we looked at structures of hTIM-1 homologues. In mTIM-1 structure, as the FG loop moves forward towards the BC loop, the $F G$ and $B C$ loops are connected by the Pro and Arg residues, forming a channel (Fig. 4). For hTIM-3, the longer strands of $\beta C$ and $\beta G$ approach to each other, so that the $F G$ and $\mathrm{BC}$ loops are too close to form a groove, whilst, a fully negatively charged valley is formed by BC loop and C'C" loop (Fig. 4). On the contrary, the BC loop of MTIM-3 extends away from the FG by up to $11.3 \AA$, leading to a flat and broader groove with negatively charged decoration on one side (Fig. 4). As to hTIM-4 and MTIM-4, three extra residues from the BC loop fill in the gap between the FG and BC loop (Fig. 4). Thus, the surface characteristics; in particular, the putative ligand-binding groove of hTIM-1 are different from those of hTIM-1 homologues. This could possibly explain why only hTIM-1 and not other TIM proteins bind EBOV GP.

\section{Multiple sites contribute to the binding of hTIM-1 to Ebola GP}

Given that hTIM-1 is the sole target among TIM family to interact with Ebola GP, at least one of three loops (the BC, $\mathrm{CC}^{\prime}$ and $\mathrm{FG}$ loops) are expected to participate in the interaction with Ebola GP. This inference is based on the 


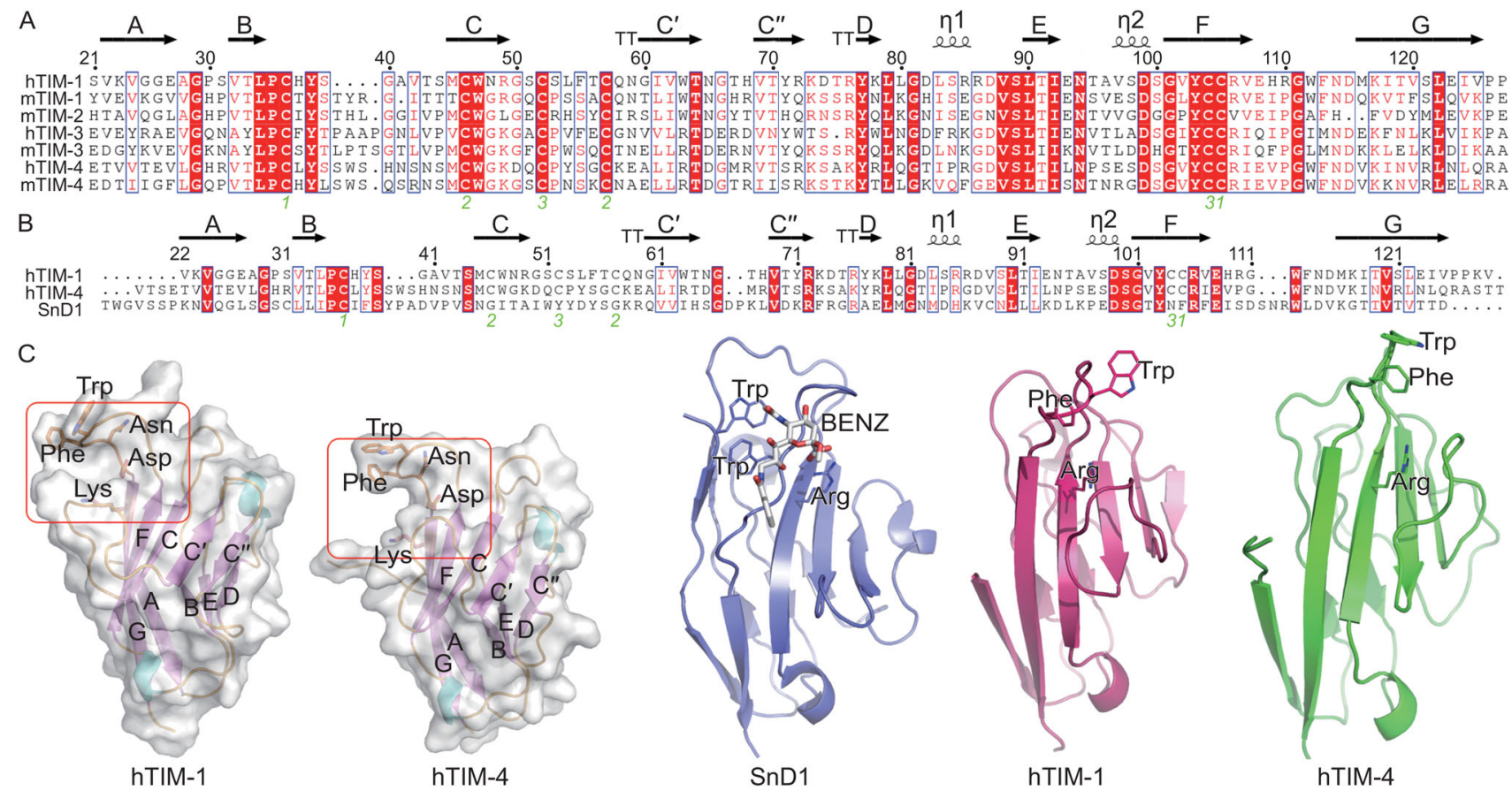

Figure 3. Sequence alignment of hTIM-1 homologues and overall structures of $\mathbf{l g}$ V domains of hTIM-1 and hTIM-4. (A and B) Amino acid sequences of hTIM-1, hTIM-3, hTIM-4, mTIM 1-4 Ig V domains and hTIM-1, hTIM-4, sialecsialo adhesin (SnD1), respectively were aligned with the program Clustal W (Larkin et al. 2007). Cys residues and disulfide bonds are marked with green numbers. (C) Ribbon diagram of hTIM-1 and hTIM- $4 \mathrm{lg}$ V domain structures. $\beta$ strands are represented in magenta and labelled with capital characters, $\alpha$ helices and loops are colored in cyan and orange respectively. A cleft responsible for PS binding is marked with a red box, critical residues for ligand binding are shown as sticks. (D) Structural comparisons of hTIM-1 Ig V with hTIM-4 Ig V and SnD1 N-terminal Ig-like domain [PDB code: 1OD9] (Zaccai et al., 2003). Binding residues of SnD1 to sialated glycoconjugates and ligand (BENZ) are shown as sticks. Potential binding residues of hTIM-1 and hTIM-4 to sialated carbohydrates are also represented as sticks.

structural analysis between $\mathrm{hTIM}-1$ and other TIM family proteins (Fig. 4) where the loops are involved in formation of cavities for ligand binding. A series of chimeras of hTIM-1 that binds EBOV GP and hTIM-4 that does not bind EBOV $\mathrm{GP}$, where corresponding structural elements were exchanged, were generated to map the region(s) that are important for Ebola GP binding (Fig. S3 and See MATERIALS AND METHODS). In the T14 (BC) mutant, for example, the BC loop of $\mathrm{hTIM}-1$ was replaced with the corresponding region of hTIM-4. We hypothesized that the binding affinity of EBOV GP to hTIM-1 would be lowered by replacement of this important region with the corresponding region from hTIM-4 and vice versa. We produced these chimeric mutants using a similar strategy as that used for producing hTIM-1 and verified them as functional units by thermal stability assays (Fig. S4). The binding affinities of EBOV GP to these chimeras were determined by SPR. As expected, the T14 (BCCC'-FG) chimera showed the most dramatic loss in binding activity to GP, whilst the T41 (BC-CC'-FG) chimera restored the binding activity to GP (Table 1 and Fig. S5). Remarkably, the T14 (FG) chimera exhibited only a slightly lower affinity for Ebola GP, while, both the T14 (BC) and T14 (CC') chimeras decreased the binding affinity by 10 folds (Table 1 and Fig. S5). In agreement with hTIM-1 chimeras, the T41 (BC$\mathrm{CC}^{\prime}$ ) and T41 (CC'-FG) chimeras gained some ability of binding to GP, but with a lower affinity of 73 and $208 \mu \mathrm{mol} / \mathrm{L}$, respectively (Table 1 and Fig. S5). An interesting observation coming out of the studies using chimeras was that exchanging FG loops did not significantly reduce the binding affinity. This can be explained from the fact that except for Arg110, all other residues of FG loop from hTIM-1 and hTIM4 are highly conserved. However, it should be noted that no significant change in binding affinity for FG loop chimeras does not necessarily mean that FG loop is not critical for Ebola GP binding. To address this issue, we constructed point mutants of residues from the FG loop of hTIM-1.

Single Ala substitutions of the 4 residues (W112/A112 or F113/A113 or N114/A114 or D115/A115) of the FG loop abolished the binding of hTIM-1 to Ebola GP (Table 1 and Fig. S5). The results are consistent with that in the case of the PS binding, all the four residues of WFND of the FG loop have been shown to be crucial for binding the ligand (Santiago 

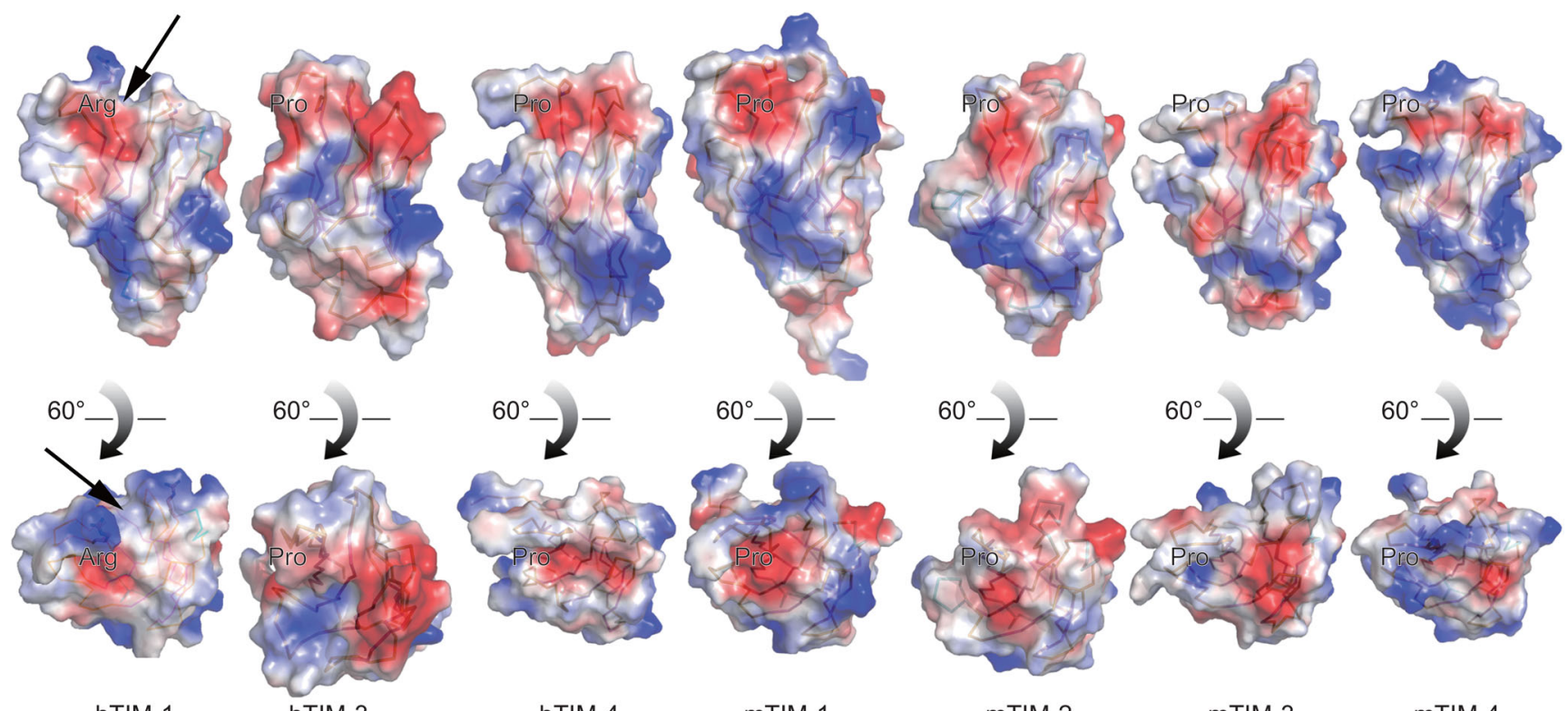

hTIM-1

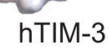

mTIM-1

mTIM-2

mTIM-3

mTIM-4

Figure 4. Electrostatic surface features relevant to the cleft for substrate binding. Distribution of charge on the surface of lg $\mathrm{V}$ of TIM family proteins is shown [PDB code: $20 R 8$ (Santiago et al., 2007), $20 R 7$ (Santiago et al., 2007), 2OYP (Cao et al., 2007), 3BI9 (Santiago et al., 2007) and 4QYC (Huang et al., 2015)]. Blue color represents positive charge; red colour, negative. A unique groove formed by the BC and FG loops of hTIM-1 is indicated by a black arrow. Arg located on the FG loop in hTIM-1 and the equivalent residue (Pro) in other TIM family members is labelled.

Table 1. Binding affinity measurements of hTIM-1 lg V mutants to EBOV GP

\begin{tabular}{lll}
\hline Mutation & Location & Affinity \\
\hline hTIM-1 & - & $2.67 \times 10^{-5}$ \\
R86A & DE loop & $5.11 \times 10^{-5}$ \\
R106A & F strand & $3.92 \times 10^{-4}$ \\
R110A & FG loop & $4.95 \times 10^{-5}$ \\
W112A & FG loop & - \\
F113A & FG loop & - \\
N114A & FG loop & $4.01 \times 10^{-4}$ \\
D115A & FG loop & $8.65 \times 10^{-3}$ \\
K117A & G strand & $1.96 \times 10^{-4}$ \\
R106A/K117A & - & - \\
T14 (BC) & - & - \\
T14 (CC') & - & - \\
T14 (FG) & - & $5.79 \times 10^{-5}$ \\
T41 (BC-CC') & - & $7.32 \times 10^{-5}$ \\
T41 (CC'-FG) & - & $2.08 \times 10^{-4}$ \\
T14 (BC-CC'-FG) & - & - \\
T41 (BC-CC'-FG) & - & $7.24 \times 10^{-5}$ \\
\hline
\end{tabular}

Binding affinity is assessed by SPR.

"-" means no detectable binding signals. et al., 2007). Two positively charged residues Arg 106 and Lys 117 in hTIM-1, which are conserved in the TIM family, are speculated to interact with the carboxylate group of the PS or sialic carbohydrate (Fig. 3C and 3D), while, these two residues were verified to play a key role in the interaction with Ebola GP as well. Single mutation of these two residues (R106/A106 or K117/A117) decreased binding of hTIM-1 to Ebola GP by $\sim 90 \%$ and double mutations (R106K117) A106A117) lost completely the binding ability (Table 1 and Fig. S5). In addition, the single mutations R86/A86 and R110/ A 110 , exhibiting $60 \%$ and $50 \%$ reduction, respectively, in the binding affinity of hTIM-1 to Ebola GP, confirmed the structural interpretation that the unique groove in $\mathrm{hTIM}-1$ also participates in GP binding (Table 1 and Fig. S5). Based on the above results, we conclude that all the three loops, $\mathrm{BC}, \mathrm{CC}^{\prime}$ and FG, of hTIM-1 together constitute the binding sites to Ebola GP, of which the residues Arg 106, Trp 112, Phe 113, Asn 114, Asp 115 and Lys 117 are critically important for Ebola GP binding, while, the residues Arg 86, Arg 110 have moderate effects on GP binding.

\section{hTIM-1 mediated Ebola pseudovirus entry integrates} multiple factors

Integration of viral GP into surrogate viruses (VSV or HIV pseudotypes) has allowed the EBOV entry to be studied in a biosafety level 2 setting. hTIM-1 was reported to promote 

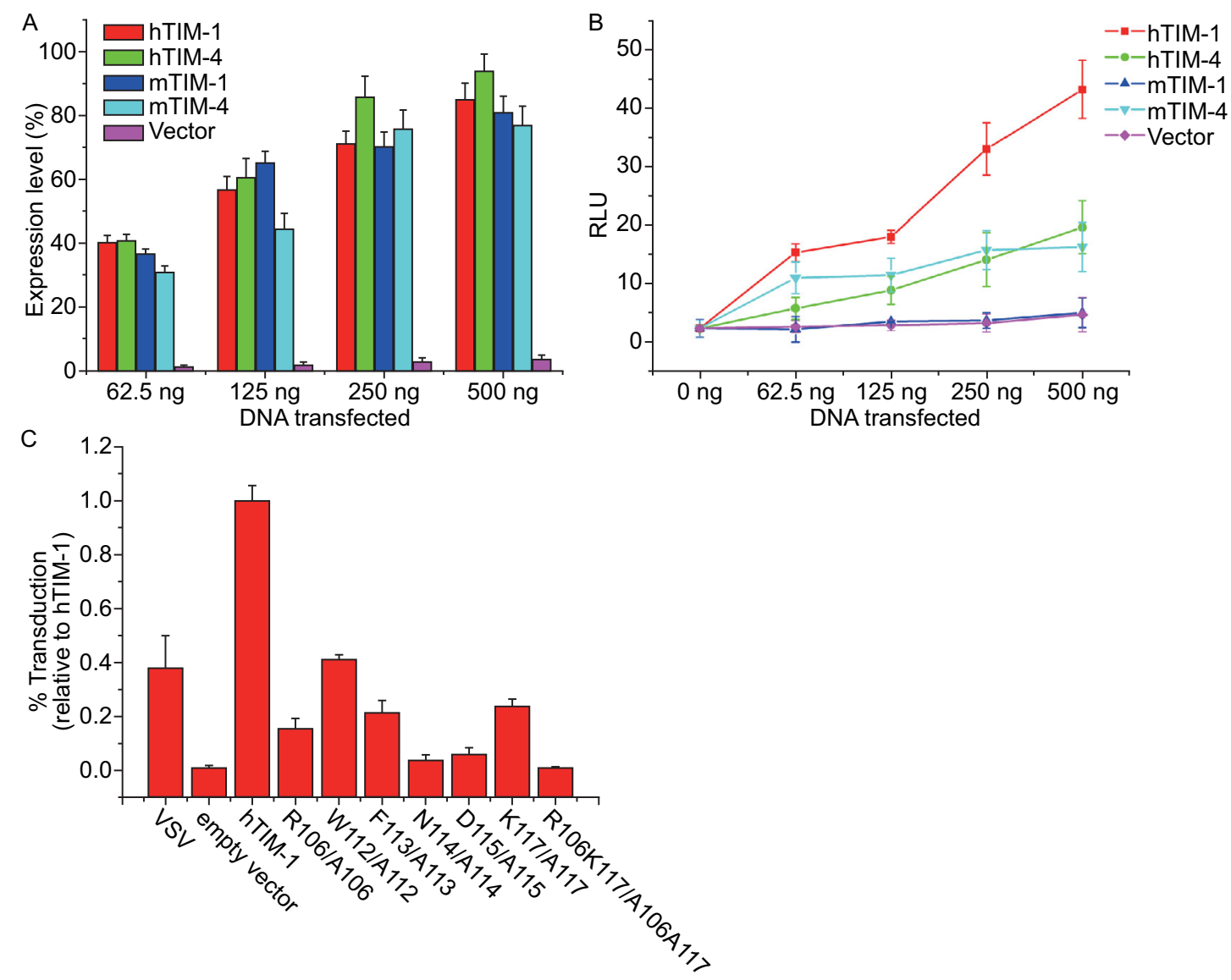

Figure 5. hTIM-1 serves as a dual-attachment receptor for EBOV and identification of hTIM-1 Ig V residues that impact EBOV GP-dependent entry. (A) Surface expression of hTIM-1, hTIM-4, mTIM-1 and mTIM-4 at $48 \mathrm{~h}$ following transfection. HEK 293T cells were transfected with increasing amounts of TIM expression plasmids or empty plasmids (62.5 ng, $125 \mathrm{ng}, 250 \mathrm{ng}$ and $500 \mathrm{ng}$ ), the expressions of TIM proteins were assessed by flow cytometry using anti-flag antibodies. (B) Compare the transduction efficiency of EBOV pseudovirus mediated by hTIM-1, hTIM-4, mTIM-1 and mTIM-4. At $48 \mathrm{~h}$, transduce EBOV pseudovirions into HEK $293 \mathrm{~T}$ cells, which were transfected with a growing amount of a hTIM-1 or hTIM-4 or mTIM-1 or mTIM-4 expression plasmid (62.5 ng, $125 \mathrm{ng}, 250$ $\mathrm{ng}$ and $500 \mathrm{ng}$ ). Luminescence signals are expressed as relative light units (RLU). (C) Identification of hTIM-1 lg V residues that impact EBOV pseudovirion entry. Compare the transduction efficiency of EBOV pseudovirions into HEK 293T cells transfected by hTIM-1 mutant constructs to that with WT hTIM-1. Empty plasmid transfected HEK 293T cells were used as a negative control. Transduction of VSV pseudovirions into HEK 293T cells is a positive control. Results shown are the mean \pm SEM of three independent experiments for panel $(\mathrm{A}-\mathrm{C})$.

some pseudoviruses entry by binding to the PS on the virion surfaces; in particular, those from the filovirus, flavivirus and alphavirus families (Jemielity et al., 2013). Although various enveloped viruses and pseudoviruses contain PS on their membrane (Mercer and Helenius, 2008), hTIM-1 is not able to promote infection of all pseudoviruses (Moller-Tank et al., 2013), which suggests that mechanisms of cellular entry differ for different viruses. To compare the ability of TIM family proteins to mediate EBOV pseudoviruses transduction systematically, we cloned hTIM-1, hTIM-4, mTIM-1 and mTIM-4 into pCMV3-SP vector with a flag tag, evaluated the surface expression of the 4 proteins in $293 \mathrm{~T}$ cells using flow cytometric assay with an anti-flag antibody (See
MATERIALS AND METHODS). Expression levels of hTIM-1, hTIM-4, mTIM-1 and mTIM-4 on the cell surface were close by transient transfection of the indicated amounts of a TIMexpressing plasmid (Fig. 5A), but hTIM-1 enhanced EBOV pseudovirus entry significantly greater than hTIM-4 and mTIM-4 (>3-fold when transfected with $0.5 \mu \mathrm{g}$ ) (Fig. 5B). Surprisingly, expression of mTIM-1 did not enhance EBOV pseudovirus transduction. Together with the fact that hTIM-3 only enhanced EBOV entry very inefficiently (Kondratowicz et al., 2011; Moller-Tank et al., 2013), these indicate that the efficiency of viral entry via interaction of TIM proteins with PS of the viral membrane is low when compared to the one mediated by direct binding between hTIM-1 and GP. This 
argument is also supported by the fact that EBOV GPbearing VP40-GFP VLPs appeared to be internalized much more efficiently than those lacking GP (Jemielity et al., 2013). Meanwhile, this argument is also consisted with lower binding affinity of hTIM1 to PS than to GP (Fig. 1E). Previously PS was reported to block the entry of EBOV into hTIM1-expressing 293T cells at the concentration of $10 \mu \mathrm{mol} / \mathrm{L}$ (Jemielity et al., 2013), however, the concentration of hTIM-1 molecules on the hTIM1-expressing 293T cell surface is $10-100 \mathrm{nmol} / \mathrm{L}$ based on the expression level analysis, it is not surprising that an approximate over 100 -fold ( $\mathrm{mol} / \mathrm{mol})$ excess PS can inhibit EBOV entry at the cellular level. EBOV entry mediated by hTIM-1 results from a dual-interaction of viral GP and PS with hTIM-1 receptor. Consistent with the results of in vitro binding assays performed using purified recombinant proteins, mutants of hTIM-1 (R106/A106, W112/A112, F113/A113, N114/A114, D115/A115, K117/A117 and R106K117/A106A117), with an equivalent expression level to that of the wild type, lower deficient in facilitating cellular entry of Ebola pseudovirus due to the inability of binding of viral PS or GP to the mutant hTIM-1 receptor (Fig. 5C).

\section{DISCUSSION}

There is an increased appreciation that the PS receptors, such as TIM family proteins, play important roles in viral infection (Moller-Tank et al., 2013; Jemielity et al., 2013; Rennert, 2011). A number of lines of evidence indicate that hTIM-1 enhances infection of a variety of enveloped viruses by binding to virion-associated PS and does not require viral entry protein (Moller-Tank et al., 2013; Meertens et al., 2012; Jemielity et al., 2013), therefore hTIM-1 is generally described as an attachment factor. Contrary to this, preferable usage of hTIM-1, not other PS receptors by filoviruses and flaviviruses suggests hTIM-1 may not just function to enhance the attachment of the virion-associated PS to cell surface. Moreover, structural analysis reporting that the membrane of flaviviruses are occluded by tightly-arranged viral glycoproteins further weakens the argument that TIM-1 is an accessory protein that enhances PS-mediated viral uptake (Zhang et al., 2003a, 2003b). Lastly, hTIM-1 was recently shown to interact with NPC1 to facilitate EBOV GPmediated membrane fusion (Kuroda et al., 2015) and direct interaction in vitro between hTIM-1 Ig V and NPC1 C-loop domain was also demonstrated by SPR (data not shown), suggesting that hTIM-1 might have additional roles and not function exclusively as an attachment factor for EBOV. In this context, our studies demonstrating a direct interaction between hTIM-1, but not other TIM family proteins, and EBOV GP in vitro by SPR confers a much bigger role on hTIM-1 in cellular entry of EBOV. We show that hTIM-1 can act as a dual-attachment receptor to recruit EBOV to cell surface by interacting directly with GP and the PS on the viral envelop. Based on our results of in vitro binding affinity studies and previously reported pseudovirion assays
(Jemielity et al., 2013), the contribution of GP to mediating EBOV entry appears to be more significant than that by PS.

The structures of $\lg \mathrm{V}$ domains of hTIM-1 and hTIM-4 reveal presence of a conserved hydrophobic cavity used by the TIM proteins for binding to PS. The ligand binding site is characterized by a constrained size of the cavity and the requirement for both an acidic group and a hydrophobic group in the ligand (Fig. $3 \mathrm{C}$ ). Structural comparisons and amino acids sequence analysis with the N-terminal lg-like domain of the Siglec family suggest that sialated glycoconjugates with an acidic group at the head and a hydrophobic group at the tail might constitute new ligands for hTIM-1/hTIM-4 (Fig. 3B and 3D). Sialic acids are mainly terminal components of glycans on glycoproteins and these sialated carbohydrates are involved in numerous cellular recognition process, such as pathogens adhesion (Kelm and Schauer, 1997; Schauer and Kamerling, 1995; Angata and Varki, 2002). EBOV GP was reported to bear sialic acid residues at the termini of a small fraction of glycans (Powlesland et al., 2008). These observations coupled with the results that deglycosylated GP exhibited a $\sim 50 \%$ reduction in binding affinity to hTIM-1 (Fig. 2F), allow us to propose that sialated carbohydrates might be additional ligands of hTIM-1/hTIM-4.

We compared the amino acid sequences and structures of hTIM-1 with other TIM family proteins, and designed a series of hTIM-1-hTIM-4 chimeras and mutants to map the regions of hTIM-1 that are important for EBOV GP binding. Three key loops (BC, CC' and FG loops) of hTIM-1 participate in interaction with EBOV GP (Table 1). Amongst these, the $\mathrm{BC}$ and FG loops in hTIM-1 form an unique groove-like structure adjacent to the PS binding site, which is in-turn formed by FG and CC' loops (Figs. 3C and 4). Additionally, we further identified a number of residues on these three loops, which are critical for the EBOV GP binding (Table 1). It is interesting to note that the binding site of PS to hTIM-1 is a subset or continuation of EBOV GP binding site on hTIM-1, which explains why hTIM-1 binds to GP with a higher affinity than hTIM-1 binds to PS.

A recent study has concluded that hTIM-1 is not only involved in filovirus attachment but it also participates in efficient membrane fusion through its interaction with NPC1 in late endosomes (Kuroda et al., 2015). Interestingly, we observed that the affinity between hTIM-1 and EBOV GP increases as the $\mathrm{pH}$ drops to that observed in endosomes $(\mathrm{pH} 7.5-5.7)$ (Fig. S6). It is possible that hTIM-1 acts as a bridge to facilitate the binding of NPC1 to GP, initializing conformational changes in GP to trigger membrane fusion.

In summary, we firstly identified the direct interaction between hTIM-1 and EBOV GP in vitro by SPR assay and solved the structures of the $\lg \mathrm{V}$ domains of hTIM-1 and hTIM-4 by crystallography, then mapped the regions in hTIM1 that are important for EBOV GP binding. Inferences gained from results of our biophysical and biochemical studies have been verified by pseudovirion infection assays. Targeting the hTIM-1-EBOV GP interaction interface to block the binding 
of EBOV GP to hTIM-1 could be a promising strategy for stalling the attachment step of Ebola virus infection.

\section{MATERIALS AND METHODS}

Structure determination

Materials, such as cells and plasmids, and methods for protein purification, crystallization and the PS binding assay can be found in the Supplementary Materials. Diffraction data sets for hTIM-1 and hTIM-4 Ig V domains were collected at beam line BL17U and BL19U of the Shanghai synchrotron facility with the highest resolution being $1.3 \AA$ and $2.3 \AA$, belonging to space groups of $P 4_{3} 2_{1} 2$ and $P 2_{1} 2_{1} 2_{1}$, respectively. Datasets were processed and scaled using the HKL2000 package (Otwinowski and Minor, 1997). One and eight protein molecules in an asymmetric unit with a solvent content of $52 \%$ and $59 \%$ (corresponding to a Matthews coefficient $V_{M}=2.55$ and $2.96 \AA^{3} \mathrm{Da}^{-1}$ (Matthews, 1968)) were found from crystals of hTIM-1 and hTIM-4, respectively. The initial structure solutions of hTIM-1 and hTIM-4 were obtained by molecular replacement using the program Phaser v2.1 (McCoy et al., 2007) with the crystal structure of mTIM-1 (Protein Data Bank [PDB] entry: 2OR8 (Santiago et al., 2007)) and hTIM-1 respectively as a search template. Manual model building and refinement were performed using COOT (Emsley and Cowtan, 2004) and PHENIX (Adams et al., 2010). The r.m.s. deviations between the eight NCS-related subunits of hTIM-4 are less than $0.12 \AA$. Chain A from hTIM- 4 was selected for following structural analysis. Structural figures were drawn with the program PyMOL (DeLano, 2002).

\section{Flow cytometric assay}

For the surface expression of hTIM-1, hTIM-4, mTIM-1 and mTIM-4, the coding sequences which start from right behind the signal peptide and last to the end were cloned into the pCMV3-SP-N-FLAG vector. So that the proteins expressed on the cell surface all have a flag tag linking at the $\mathrm{N}$ terminal. The plasmids were transfected into TIM1 $\%$ 293T cells using lipofectamine2000 according to the manufacturer's instructions (Invitrogen). The cells were collected $48 \mathrm{~h}$ after transfection. To measure the expression, cells were stained with PE anti-flag antibody and with PE rat Ig G2a antibody as negative control (Biolegend). 7-AAD was used to monitor the dead cells through binding to the nucleic acid. The data was analyzed using the Summit v4.3 software.

\section{Surface plasmon resonance assay}

The BIAcore experiments were carried out at room temperature $\left(25^{\circ} \mathrm{C}\right)$ using a BIAcore 3000 machine with $\mathrm{CM} 5$ chips. For all the measurements, a PBST buffer consisting of PBS, pH 7.4 and $0.005 \%(v / v)$ Tween-20 was used. The Ebola GP proteins were immobilized on the chip at about 7000 response units. Gradient concentrations of human TIM1 and its mutations (0, 0.625, 1.25, 2.5, $5,10,20,40 \mu \mathrm{mol} / \mathrm{L}$ ) were then used to flow over the chip surface. The binding kinetics were analysed with the software BIAevaluation Version 4.1 using the steady state affinity. To study the effect of the ligand $\mathrm{PS}$ and $\mathrm{pH}$ on the binding of hTIM-1 to EBOV GP, the concentration of hTIM-1 was maintained constant (10 $\mu \mathrm{mol} / \mathrm{L})$. The concentration of PS was gradually increased (PS: $200 \mu \mathrm{mol} / \mathrm{L}$, $500 \mu \mathrm{mol} / \mathrm{L}, 1 \mathrm{mmol} / \mathrm{L}, 2 \mathrm{mmol} / \mathrm{L}$ ) in the binding buffer. Similarly, the buffer $\mathrm{pH}$ was gradually changed from 7.5 to 5.7 in steps of 0.3 units. The response values were recorded to analyze the changes.

\section{Thermofluor assay}

Thermofluor experiments were performed with iCycleriQ Real Time PCR Detection System (Bio-Rad) instrument. SYPRO orange was used as fluorescent probe to monitor the denaturation of the proteins. $25 \mu \mathrm{L}$ reactions were set up in a PCR plate, containing $0.1 \mathrm{mg} / \mathrm{mL}$ each protein, $5 \times$ Sypro Orange in $\mathrm{pH}$ ranging from 4.0 to 8.4 buffer solutions and ramped from $20-95^{\circ} \mathrm{C}$ with fluorescence recorded in triplicate at $1^{\circ} \mathrm{C}$ intervals.

\section{Pseudovirion}

Pseudovirions bearing Ebola virus GP protein were produced in 293T cells transfected with PNL 4-3 vector containing Luciferase as a reporter and pcDNA3.1 vector, which contains GP, using lipofectamine2000 according to the manufacturer's instructions. Pseudovirus-containing culture supernatant was harvested at $48 \mathrm{~h}$ posttransfection and stored at $-80^{\circ} \mathrm{C}$.

For infection, cells were plated on $96-$ well plates and incubated at $37^{\circ} \mathrm{C}$ with serially diluted pseudovirus-containing supernatant to yield proper level of infection. Supernatants were replaced with fresh medium after $3 \mathrm{~h}$ of incubation post infection by pseudovirus. Another $48 \mathrm{~h}$ is needed to allow for luciferase reporter to expression and infection levels were assessed by measuring bioluminescence signals.

\section{Analytical ultracentrifugation}

Sedimentation velocity experiments were performed on a Beckman $\mathrm{XL}-\mathrm{I}$ analytical ultracentrifuge at $20^{\circ} \mathrm{C}$. Protein samples were diluted with lysis buffer to $400 \mu \mathrm{L}$ at an $A_{280 \mathrm{~nm}}$ absorption of about 0.8 . Samples were loaded into a conventional double-sector quartz cell and mounted in a Beckman four-hole An-60 Ti rotor. Data were collected at $60,000 \mathrm{rpm}$ at a wavelength of $280 \mathrm{~nm}$. Interference sedimentation coefficient distributions were calculated from the sedimentation velocity data using the SEDFIT software program (www.analyticalultracentrifugation.com).

\section{ACKNOWLEDGEMENTS}

We thank Neil Shaw for assistance with the manuscript and advice, Xiaoxia Yu, Yuanyuan Chen and Ya Wang provided expert assistance. We gratefully acknowledge the assistance of the staff of the beamline BL17U and BL19U at Shanghai Synchrotron with the X-ray diffraction data collection. We also thank Core Facility of the Institute of Biophysics, Chinese Academy of Sciences (CAS). Work was supported by the National Basic Research Program (973 Program) (No. 2014CB542800), the National Natural Science Foundation of China (Grant Nos. 81330036, 31570717 and 81520108019) and the Strategic Priority Research Program of the Chinese Academy of Sciences, Grant No. XDB08020200. 


\section{ABBREVIATIONS}

EBOV, Ebolavirus; GP, glycoprotein; hTIM-1, human T-cell immunoglobulin and mucin domain protein 1; MLD, mucin-like domain; RBD, receptor binding domain.

\section{COMPLIANCE WITH ETHICS GUIDELINES}

Shuai Yuan, Lei Cao, Hui Ling, Minghao Dang, Yao Sun, Xuyuan Zhang, Yutao Chen, Liguo Zhang, Dan Su, Xiangxi Wang and Zihe Rao declare that they have no conflict of interest.

This article does not contain any studies with human or animal subjects performed by the any of the authors.

\section{AUTHOR CONTRIBUTIONS}

X.W. and Z.R. designed research; S.Y., L.C., H.L., M.D., Y.S., X.Z. and Y.C. performed research; S.Y., X.W. and Z.R. analyzed data and wrote the manuscript.

\section{ACCESSION CODES}

Coordinates and structure factors have been deposited with RCSB accession codes: 5DZN, 5DZO.

\section{OPEN ACCESS}

This article is distributed under the terms of the Creative Commons Attribution 4.0 International License (http://creativecommons.org/ licenses/by/4.0/), which permits unrestricted use, distribution, and reproduction in any medium, provided you give appropriate credit to the original author(s) and the source, provide a link to the Creative Commons license, and indicate if changes were made.

\section{REFERENCES}

Adams PD et al (2010) PHENIX: a comprehensive python-based system for macromolecular structure solution. Acta Crystallogr Sect D 66(2):213-221

Angata T, Varki A (2002) Chemical diversity in the sialic acids and related $\alpha$-keto acids: an evolutionary perspective. Chem $\operatorname{Rev} 102$ (2):439-470

Brindley MA et al (2007) Ebola virus glycoprotein 1: identification of residues important for binding and postbinding events. J Virol 81 (14):7702-7709

Cao E et al (2007) T cell immunoglobulin mucin-3 crystal structure reveals a galectin-9-independent ligand-binding surface. Immunity 26(3):311-321

Côté $M$ et al (2011) Small molecule inhibitors reveal Niemann-Pick C1 is essential for Ebola virus infection. Nature 477(7364):344348

DeLano WL (2002) The PyMOL molecular graphics system

Dolnik O, Kolesnikova L, Becker S (2008) Filoviruses: interactions with the host cell. Cell Mol Life Sci 65(5):756-776

Emsley P, Cowtan K (2004) Coot: model-building tools for molecular graphics. Acta Crystallogr Sect D 60(12):2126-2132
Feigelstock D, Thompson P, Mattoo P, Zhang Y, Kaplan GG (1998) The human homolog of $\mathrm{HAV}$ cr-1 codes for a hepatitis $A$ virus cellular receptor. J Virol 72(8):6621-6628

Freeman GJ, Casasnovas JM, Umetsu DT, DeKruyff RH (2010) TIM genes: a family of cell surface phosphatidylserine receptors that regulate innate and adaptive immunity. Immunol Rev 235(1):172-189

Huang $\mathrm{Y}-\mathrm{H}$ et al (2015) CEACAM1 regulates TIM-3-mediated tolerance and exhaustion. Nature 517(7534):386-390

Hunt CL, Lennemann NJ, Maury W (2012) Filovirus entry: a novelty in the viral fusion world. Viruses 4(2):258-275

Jemielity $S$ et al (2013) TIM-family proteins promote infection of multiple enveloped viruses through virion-associated phosphatidylserine. PLoS Pathog 9(3):e1003232

Kelm S, Schauer R (1997) Sialic acids in molecular and cellular interactions. Int Rev Cytol 175:137-240

Kondratowicz AS et al (2011) T-cell immunoglobulin and mucin domain 1 (TIM-1) is a receptor for Zaire Ebolavirus and Lake Victoria Marburgvirus. Proc Natl Acad Sci 108(20):8426-8431

Kuchroo VK, Umetsu DT, DeKruyff RH, Freeman GJ (2003) The TIM gene family: emerging roles in immunity and disease. Nat Rev Immunol 3(6):454-462

Kuhn JH et al (2006) Conserved receptor-binding domains of Lake Victoria marburgvirus and Zaire ebolavirus bind a common receptor. J Biol Chem 281(23):15951-15958

Kuroda M, Fujikura D, Nanbo A, Marz A, Noyori O, Kajihara M, Maruyama J, Yoshida R, Feldmann H, Takada A (2015) The interaction between TIM-1 and NPC1 is important for the cellular entry of Ebola virus. J Virol 89:6481

Larkin M et al (2007) Clustal W and Clustal X version 2.0. Bioinformatics 23(21):2947-2948

Lee JE et al (2008) Structure of the Ebola virus glycoprotein bound to an antibody from a human survivor. Nature 454(7201):177-182

Li M et al (2014) TIM-family proteins inhibit HIV-1 release. Proc Natl Acad Sci 111(35):E3699-E3707

Matthews BW (1968) Solvent content of protein crystals. J Molbiol 33(2):491-497

May A, Robinson R, Vinson M, Crocker P, Jones E (1998) Crystal structure of the $\mathrm{N}$-terminal domain of sialoadhesin in complex with $3^{\prime}$ sialyllactose at $1.85 \AA$ resolution. Mol Cell 1(5):719-728

McCoy AJ et al (2007) Phaser crystallographic software. J Appl Crystallogr 40(4):658-674

Meertens L et al (2012) The TIM and TAM families of phosphatidylserine receptors mediate dengue virus entry. Cell Host \& Microbe 12(4):544-557

Mercer J, Helenius A (2008) Vaccinia virus uses macropinocytosis and apoptotic mimicry to enter host cells. Science $320(5875)$ : 531-535

Moller-Tank S, Kondratowicz AS, Davey RA, Rennert PD, Maury W (2013) Role of the phosphatidylserine receptor TIM-1 in enveloped-virus entry. J Virol 87(15):8327-8341

Otwinowski Z, Minor W (1997) Processing of X-ray diffraction data. Methods Enzymol 276:307-326

Powlesland AS et al (2008) A novel mechanism for LSECtin binding to Ebola virus surface glycoprotein through truncated glycans. J Biol Chem 283(1):593-602

Rennert PD (2011) Novel roles for TIM-1 in immunity and infection. Immunol Lett 141(1):28-35 
Santiago C et al (2007a) Structures of T cell immunoglobulin mucin receptors 1 and 2 reveal mechanisms for regulation of immune responses by the TIM receptor family. Immunity 26(3):299-310

Santiago C et al (2007b) Structures of T cell immunoglobulin mucin protein 4 show a metal-lon-dependent ligand binding site where phosphatidylserine binds. Immunity 27(6):941-951

Schauer R, Kamerling JP (1995) Chemistry, biochemistry and biology of sialic acids. New Compr Biochem 29:243-402

Silberstein E et al (2003) Alteration of hepatitis A virus (HAV) particles by a soluble form of HAV cellular receptor 1 containing the immunoglobulin-and mucin-like regions. J Virol 77(16):8765-8774

Soares MM, King SW, Thorpe PE (2008) Targeting inside-out phosphatidylserine as a therapeutic strategy for viral diseases. Nat Med 14(12):1357-1362

Takada A et al (1997) A system for functional analysis of Ebola virus glycoprotein. Proc Natl Acad Sci 94(26):14764-14769
Wang $X$ et al (2014) Hepatitis A virus and the origins of picornaviruses. Nature

Wool-Lewis RJ, Bates P (1998) Characterization of Ebola virus entry by using pseudotyped viruses: identification of receptor-deficient cell lines. J Virol 72(4):3155-3160

Zaccai NR et al (2003) Structure-guided design of sialic acid-based Siglec inhibitors and crystallographic analysis in complex with sialoadhesin. (Translated from eng). Structure 11(5):557-567 (in eng)

Zhang $W$ et al (2003a) Visualization of membrane protein domains by cryo-electron microscopy of dengue virus. Nat Struct Mol Biol 10(11):907-912

Zhang $Y$ et al (2003b) Structures of immature flavivirus particles. EMBO J 22(11):2604-2613 